\title{
Release behavior of 2,4-dichlorophenoxyacetic acid herbicide using novel porous polyacrylamide hydrogels
}

\author{
Gholam Reza Mahdavinia, ${ }^{1 *}$ Seyyed Bahman Mousavi, ${ }^{2}$ Farrokh Karimi, ${ }^{3}$ Gholam \\ Bagheri Marandi, ${ }^{4}$ Saleh Shahabivand, ${ }^{3}$ Mohammad Harati ${ }^{5}$ \\ ${ }^{1 *}$ Department of Chemistry, Faculty of Science, University of Maragheh, P.O. Box \\ 55181-83111, Maragheh, Iran; fax: +98-421-2276060; tel: +98-421-2273068; e-mail: \\ grmnia@mhec.ac.ir, \\ ${ }^{2}$ Department of Soil Science, Faculty of Agriculture, University of Maragheh, P.O. Box \\ 55181-83111, Maragheh, Iran. \\ ${ }^{3}$ Department of Biotechnology, Faculty of Science, University of Maragheh, P.O. Box \\ 55181-83111, Maragheh, Iran. \\ ${ }^{4}$ Department of Chemistry, Islamic Azad University, Karaj Branch, P. O. Box 31485- \\ 313, Karaj, Iran. \\ ${ }^{5}$ Department of Chemistry, University of Western Ontario, London, ON N6A 5B7, \\ Canada.
}

(Received: 23 September, 2008; published: 26 December, 2009)

\begin{abstract}
Porous polyacrylamide (PAAm) was synthesized using calcium carbonate $(\mathrm{CCb})$ micro-powder and subsequent leaching process. Polymerization was carried out in a solution environment system containing methylenebis acrylamide (MBA) and acrylamide (AAm) as crosslinker and monomer, respectively. $\mathrm{CCb}$ micro-powders were introduced to the polymerization solution before adding initiator. Ammonium persulfate/sodium metabisulfite (APS/SMBS) were used as a redox initiator system. $\mathrm{HCl}$ solution was used to remove $\mathrm{CCb}$ powders. The effect of MBA concentration and CCB amount on the swelling of hydrogels were studied. The porous structure of hydrogels was investigated and verified using scanning electron microscopy (SEM). 2,4-Dichlorophenoxyacetic acid $(2,4-D)$ was loaded into the hydrogels and release of this herbicide was investigated. The effect of MBA concentration and addition of $\mathrm{CCb}$ on the content of released herbicide was studied. Also, the study of herbicide release in a solution under various $\mathrm{pH}$ revealed the $\mathrm{pH}$-dependency release of 2,4-D herbicide.

Keywords: Polyacrylamide, Porous, Hydrogel, Herbicide, Release
\end{abstract}

\section{Introduction}

Hydrogels are three-dimensional crosslinked hydrophilic polymer networks, which swell without dissolving when brought into water or biological fluids [1]. These crosslinked polymers have been used widely in various types of applications such as controlled drug delivery, immobilization of enzymes, dewatering of protein solution, solute separation, baby diapers, soil for agriculture and horticulture, water-blocking tape, absorbent pads, and others [2-8]. There are several well known methods to prepare porous hydrogels, including phase separation, foaming technique, emulsiontemplate synthesis, and particulate leaching and freeze-drying [10, 11]. Park et al. developed a new kind of superabsorbent polymers, so called superporous hydrogels [9]. Scientists have been studying porous PAAm hydrogels and their derivatives as important hydrophilic polymers for preparing hydrogels [12-17]. Abd El-Rehim and 
coworkers $[18,19]$ investigated applications of PAAm hydrogels in controlled release of agrochemicals and bioactive materials.

The delivery of herbicides by controlled release formulation offers both ecological and economical advantages. Success of these formulations is based on suitable choices of polymer support [20]. Controlled release of various herbicides such as simazine, 2,4,5-trichlorophenoxyacetic acid, triazine, and fenuron by using polymeric matrixes have been subject of numerous studies [21-24]. Herbicides have been extensively employed; for example, the herbicide 2,4-D has been used for a long time in agriculture, forestry, and home and garden application and to control weeds in parks for over 50 years [25]. Polymeric matrixes such as cornstarch, starch-g-poly(butyl acrylate) and alginate gels have been used for controlled release of 2,4-D [26-28].

Synthesizing porous hydrogels using solution polymerization method (with low concentration of monomers) in the presence of porogen (gas bubbles are produced from reaction of porogen with chemicals) is a difficult task, because produced gas bubbles from porogen leave the solution easily. It is well known that calcium carbonate is poorly soluble in neutral or basic media; however, it reacts with acid and produces carbon dioxide. To the best of our knowledge, there has been no previous report on using the calcium carbonate to synthesize porous PAAm hydrogel. The leaching of calcium carbonate and subsequent evolution of $\mathrm{CO}_{2}$ gas from a hydrogel matrix results in porous structure. The major part of this report is focused on synthesizing porous PAAm hydrogel through solution polymerization and its application on release of 2,4-D. This herbicide was loaded in the purified hydrogel and the release of this active agent in deionized water was studied.

\section{Results and Discussion}

\section{Synthesis and Characterization}

We have successfully prepared porous PAAm hydrogel using solution polymerization with a particulate leaching technique and subsequent releasing of 2,4-D have been studied. Preparing porous hydrogels via porogen method in solution with low concentration of monomers is a challenging task as bubbles may leave the solution without having chance to make the hydrogel porous. Incorporation of insoluble material such as $\mathrm{CaCO}_{3}$ in the polymer composition and subsequently their leaching is a suitable method to make a porous polymer. The porous structure can be resulted in two ways: (a) acid treatment to leach the $\mathrm{CaCO}_{3}$ particles and (b) trapping evolved $\mathrm{CO}_{2}$ gas, from neutralization of $\mathrm{CaCO}_{3}$ in the presence of acid, in highly pasty and jelly state hydrogel and as the result, released gas creates pores in the hydrogel structure.

Figure 1 shows typical SEM micrographs of prepared hydrogels. The PAAm hydrogel and $\mathrm{CaCO}_{3}$ particles are observable in the hybrids, as shown in Fig. $1 \mathrm{~b}$. After $\mathrm{HCl}$ treatment, the $\mathrm{CaCO}_{3}$ particles had disappeared and pores could be clearly observed in the hydrogel structure, shown in Fig. 1c. On the other hand, we were not able to create pores in hydrogels prepared without the $\mathrm{CaCO}_{3}$ particles (see Fig. 1a). These observations indicate successful preparation of porous PAAm hydrogels by the present technique. 


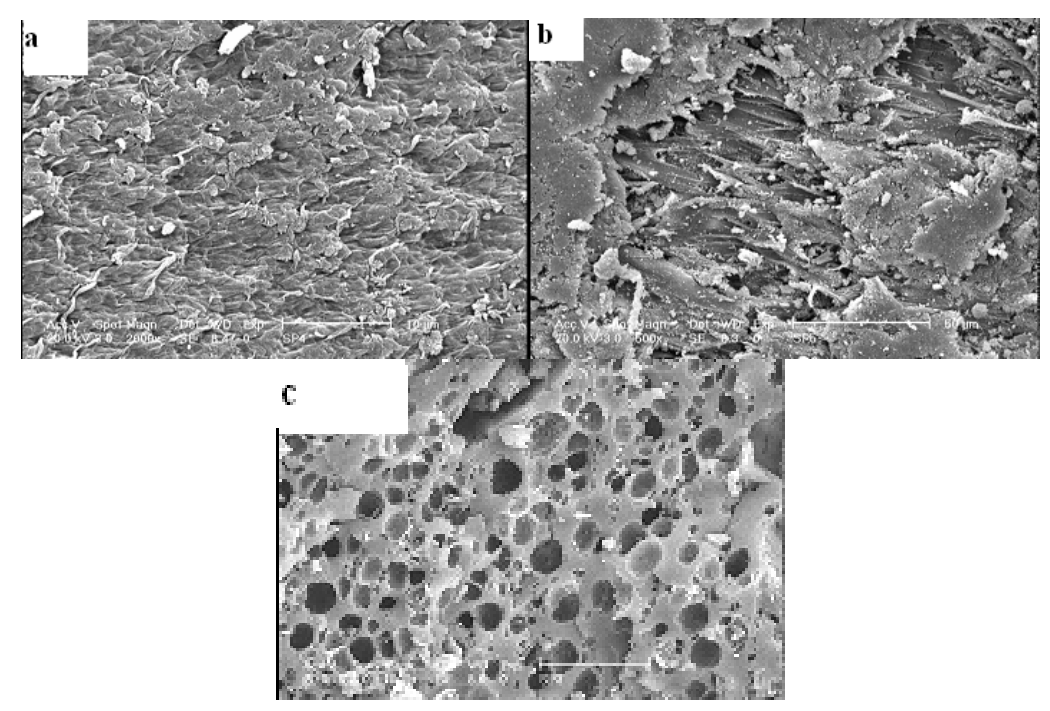

Fig. 1. SEM micrographs of (a) non-porous PAAm (b) PAAm hydrogel containing $\mathrm{CaCO}_{3}$ and (c) porous hydrogel after removing $\mathrm{CaCO}_{3}$.

\section{Effect of $\mathrm{CaCO}_{3}$ content on porosity}

Hydrogel preparation method affects the formation of micropores and macropores in hydrogels structure. When hydrogels are synthesized by conventional methods, the result will be the formation of micropores. Through introducing the porogen agent during hydrogel synthesis, not only micropores will be produced, but also macropores will exist [29]. Figure 2 depicts the structure of porous hydrogel containing micropores and macropores. In fact, the macropores are attributed to the pores that are created from leaching of $\mathrm{CCb}$ powders. While, the gaps among molecular chains in PAAm hydrogels (that may be containing wet) are assumed as micropores [30].

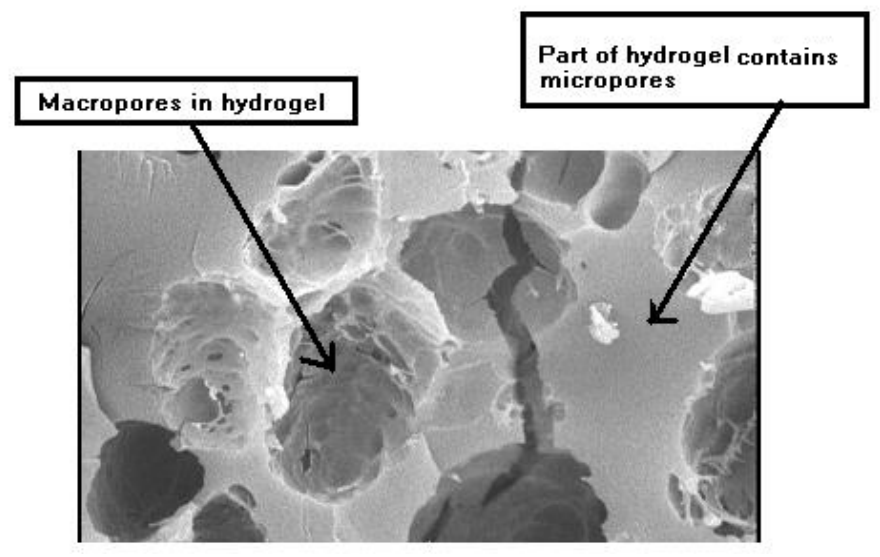

Fig. 2. Macropores and micropores in porous hydrogels.

The ratio of macropores to micropores can affect the swelling behavior of the hydrogels. So, it is important to determine this ratio using suitable method. Recently, a simple method to determine the macropores to micropores ratio in porous PAAm hydrogels has been reported by Tamagawa et al [30]. After inclusion of macropores in the PAAm hydrogel, this research group has performed no further treatment on the hydrogels. So, after synthesis of hydrogels and without drying them, by choosing a definite volume of hydrogels and comparing the volume and weight of hydrogels, the 
macropores to micropores ratio could be calculated. According to this method, we attempted to determine macropores to micropores ratio in the hydrogels. But there was a problem; in fact, the $\mathrm{CaCO}_{3}$ should be removed using $\mathrm{HCl}$ solution. During the leaching of $\mathrm{CaCO}_{3}$, the water can diffuse into produced macropores causing an error in the calculation. So, we tried to dry the hydrogels, and subsequently determine the bulk density of the dried hydrogels.

For all hydrogels, the bulk density of dried hydrogels was determined using picnometer. As shown in Table 1, the increase in $\mathrm{CaCO}_{3}$ content has a big influence on the density of the hydrogels. The results reveal that the increase in $\mathrm{CaCO}_{3}$ causes a decrease in the density of the hydrogels.

Tab. 1. Effect of $\mathrm{CaCO}_{3}$ content on the bulk density $(\rho)$ and volume fractions of macropores and micropores in dried hydrogels.

\begin{tabular}{cccccc}
\hline Sample & $\mathrm{CaCO}_{3}, \mathrm{mg} / \mathrm{mL}$ & $\rho, \mathrm{g} / \mathrm{mL}$ & $V f_{\text {macro }}$ & $V f_{\text {micro }}$ & $\left(V f_{\text {macro }}\right) /\left(V f_{\text {micro }}\right)$ \\
SP1 & 0 & 1.64 & 0 & 1 & 0 \\
SP2 & 38 & 1.23 & 0.25 & 0.75 & 0.33 \\
SP3 & 75 & 1.02 & 0.375 & 0.622 & 0.6 \\
SP4 & 150 & 0.85 & 0.48 & 0.52 & 0.9 \\
\hline
\end{tabular}

For a definite weight of dried hydrogels $\left(W_{d g}\right.$ and $W_{d g}{ }^{\circ}$, the weights of dried hydrogels prepared using $\mathrm{CaCO}_{3}$ and no- $\mathrm{CaCO}_{3}$, respectively), the difference in volumes can be attributed to the volume fraction of macropores $\left(V f_{\text {macro }}\right)$. So, we can write:

$V f_{\text {macro }}=\frac{V_{d g}-V_{d g}^{o}}{V_{d g}}$

where, $V_{d g}{ }^{\circ}$ and $V_{d g}$ are the volumes of dried hydrogels synthesized without and with $\mathrm{CaCO}_{3}$, respectively. For equal weights of dried hydrogels, the Eq (1) can be written as below:

$W=\rho V$

$V f_{\text {macro }}=\frac{\frac{W_{d g}}{\rho_{d g}}-\frac{W_{d g}^{o}}{\rho_{d g}^{o}}}{\frac{W_{d g}}{\rho_{d g}}}$

$V f_{\text {macro }}=\frac{\rho_{d g}^{o}-\rho_{d g}}{\rho_{d g}^{o}}$

It may be noted that in a hydrogel containing macropores and micropores the sum of these volume fractions will be as below:

$V f_{\text {macro }}+V f_{\text {micro }}=1$

where, $V f_{\text {macro }}$ and $V f_{\text {micro }}$ are volume fractions of macropores and micropores, respectively. From Table 1 it can be observed that the $V f_{\text {macro }}$ of hydrogels and subsequently, $\left(V f_{\text {macro }}\right) /\left(V f_{\text {micro }}\right)$ ratio increase with an increase in $\mathrm{CaCO}_{3}$ content. Figure 3 shows the SEM micrographs of hydrogels prepared with various content of $\mathrm{CaCO}_{3}$. The SEM results reveal the increase in macropores content with increasing $\mathrm{CaCO}_{3}$ content. The higher $\mathrm{CaCO}_{3}$ content causes higher number of macropores and subsequently the density of hydrogels is decreased. 


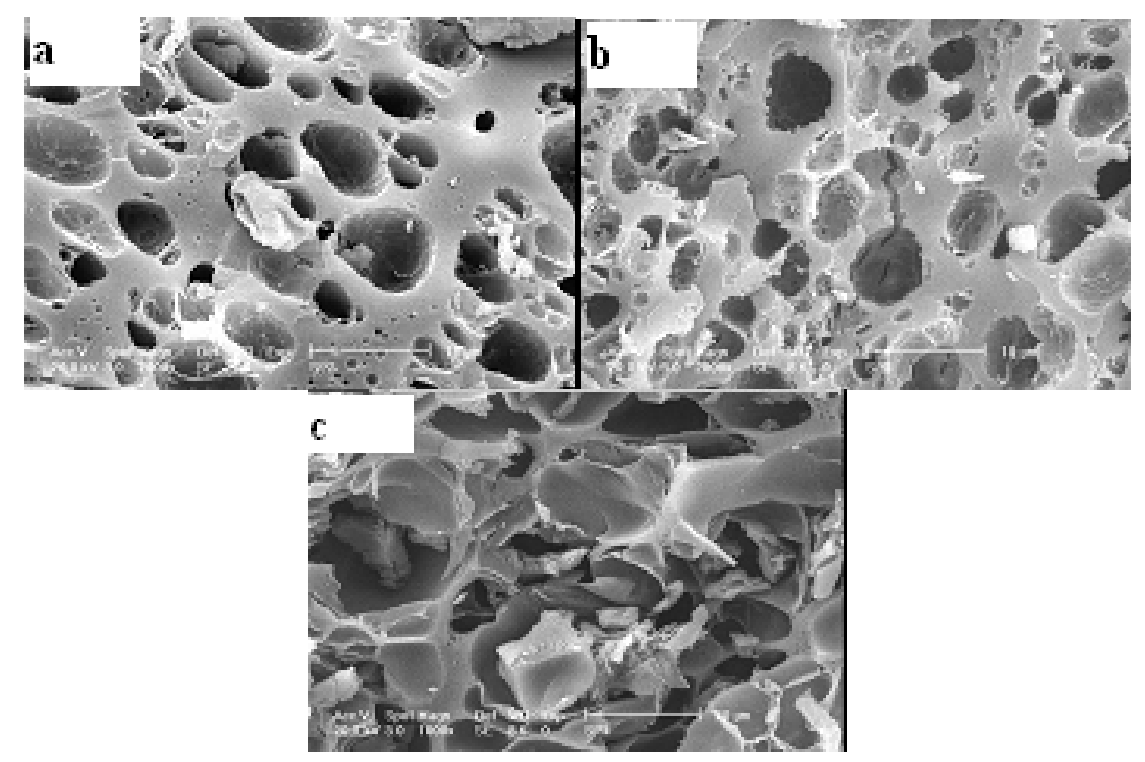

Fig. 3. Surface morphology of PAAm hydrogels using SEM for samples prepared by $38 \mathrm{mg} / \mathrm{mL}$ (a), $75 \mathrm{mg} / \mathrm{mL}$ (b) and $150 \mathrm{mg} / \mathrm{mL}$ (c) of $\mathrm{CaCO}_{3}$.

\section{Swelling Behaviour of Hydrogels}

Many factors affect the water absorbency of hydrogels. Among them, we study the effect of MBA crosslinker concentration and $\mathrm{CCb}$ amount on the swelling. Figure 4 illustrates water-absorbing dependency of hydrogels on MBA concentration. As the concentration of the MBA was increased, the water absorbency of the hydrogel was decreased (41, 34 and $17 \mathrm{~g} / \mathrm{g}$ for $0.13,0.32$ and $0.65 \mathrm{mmol}$ of MBA, respectively). Clearly, higher concentration of crosslinker produces larger degree of polymer chains branching and generates an additional network. Thereby, with increasing crosslinker content, the crosslinking density increases and subsequently the network space gets diminished and less water enters the hydrogel [31].

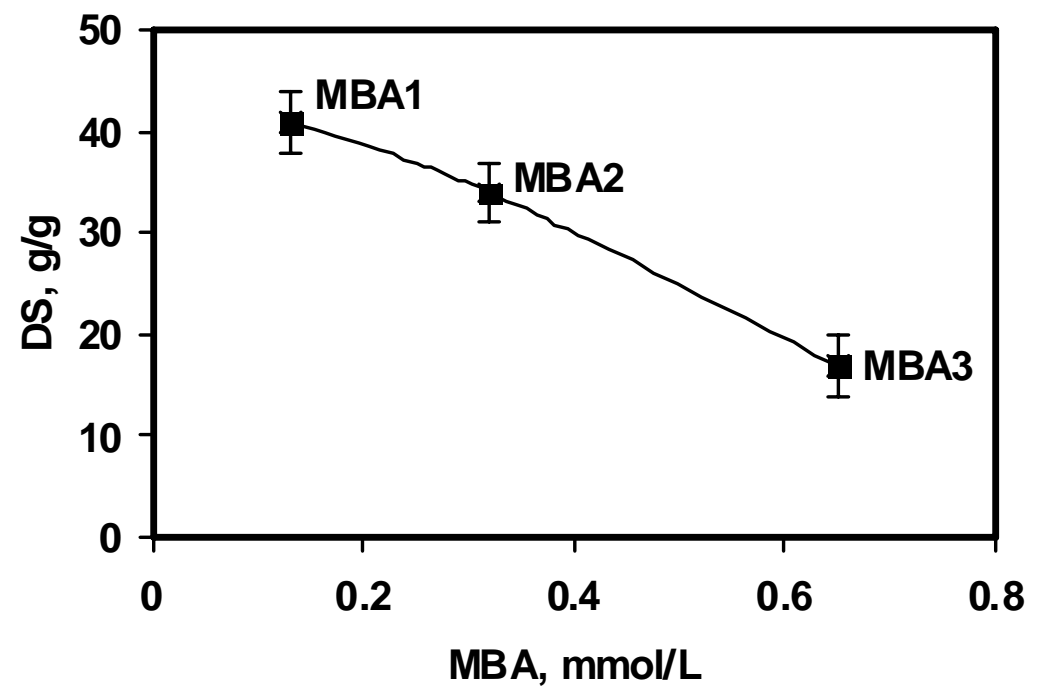

Fig. 4. Effect of MBA concentration on the water-absorbency of hydrogels. 
Figure 5 illustrates the equilibrium of water content and dynamic swelling behaviour of hydrogels as a function of the $\mathrm{CaCO}_{3}$ amount. As presented in this figure, by using the $\mathrm{CaCO}_{3}$ in the feed mixture, the water absorbency of the hydrogels increases. This may be attributed to the fact that using $\mathrm{CaCO}_{3}$ in the hydrogel makes pores in the hydrogel structure and results in a high water uptake. Also, hydrogels absorb higher amount of water with increasing $\mathrm{CaCO}_{3}$ amount in reaction feed that may be due to increase in pore number. Furthermore, we have investigated the effect of $\mathrm{CaCO}_{3}$ content on the swelling kinetic of the hydrogels. A preliminary study was conducted on the hydrogels swelling kinetics and results have been presented in Figure 5 which shows the swelling behaviour dynamics of PAAm hydrogel samples.

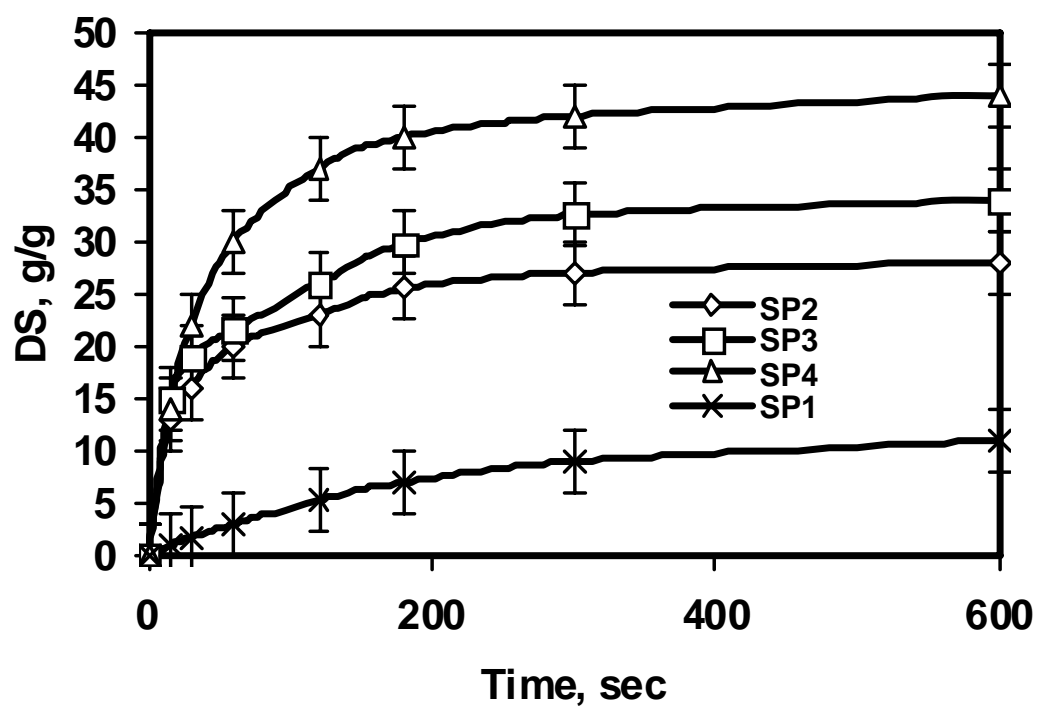

Fig. 5. Dynamic swelling behaviors of hydrogels as a function of the $\mathrm{CaCO}_{3}$ amount.

The rate of water uptake in porous hydrogels is higher than non-porous hydrogels. Increase in surface area and porosity of hydrogels amplify the rate of water absorption. The swelling-time data can be fitted with the following equation [32] to derive the power and rate parameters:

$S_{t}=S_{e}\left(1-e^{-t / \tau}\right)$

where $S_{t}$ is swelling at time $t(\mathrm{~g} / \mathrm{g}), S_{e}$ is equilibrium swelling ("power parameter", $(\mathrm{g} / \mathrm{g})$, $t$ is time (s) for swelling $S_{t}$, and $\tau$ stand for "rate parameter" (time for $S_{\tau}$ ), s. The rate parameters for hydrogels are illustrated in Table 2. The $\tau$ value for hydrogel with no$\mathrm{CaCO}_{3}$ is higher than samples which were synthesized in the presence of $\mathrm{CaCO}_{3}$ because latter type of hydrogels has higher number of macropores in their structure.

Tab. 2. Degree of swelling and $\tau$ values for hydrogels synthesized by various amount of $\mathrm{CCb}$.

\begin{tabular}{ccccc}
\hline run & SP1 & SP2 & SP3 & SP4 \\
\hline DS, g/g & 23 & 28 & 34 & 44 \\
$\tau, \mathrm{s}$ & 500 & 128 & 113 & 100 \\
\hline
\end{tabular}




\section{Release Study of 2,4-D}

Not only the degree of swelling, dynamics of swelling, and type of hydrogel affect the release of the active agent from polymeric matrix, but also dissolution and nature of the active agent may have an influence on the amount of drug released [6, 33]. In this work, PAAm as a non-ionic hydrogel is chosen to study the release of 2,4-D herbicide. The structure of this herbicide is shown in Scheme 1. As we can see from this scheme, it contains carboxylic acid group $(\mathrm{pKa}=2.6)$ which makes its solubility dependent on the solution $\mathrm{pH}$ [34]. Thus, release of the active agent was studied as a function of hydrogel porosity, crosslinker concentration, and $\mathrm{pH}$ of medium.<smiles>O=C(O)COc1ccc(Cl)cc1Cl</smiles>

2,4-Dichlorophenoxyacetic acid (2,4-D)

Scheme 1. Chemical structure of 2,4-dichlorophenoxyacetic acid.

\section{Effect of Porosity on Release of 2,4-D}

The release profiles of 2,4-D as a function of porosity of hydrogels are shown in Figure 6 . The porous nature of PAAm hydrogels has two main effects. First, increase in porosity leads to increase in the percentage of loading i.e., boost in swelling capacity of hydrogels because of raise in porosity, causes high loading of the active agent and it subsequently creates high release of it (Experimental section, Table 4).

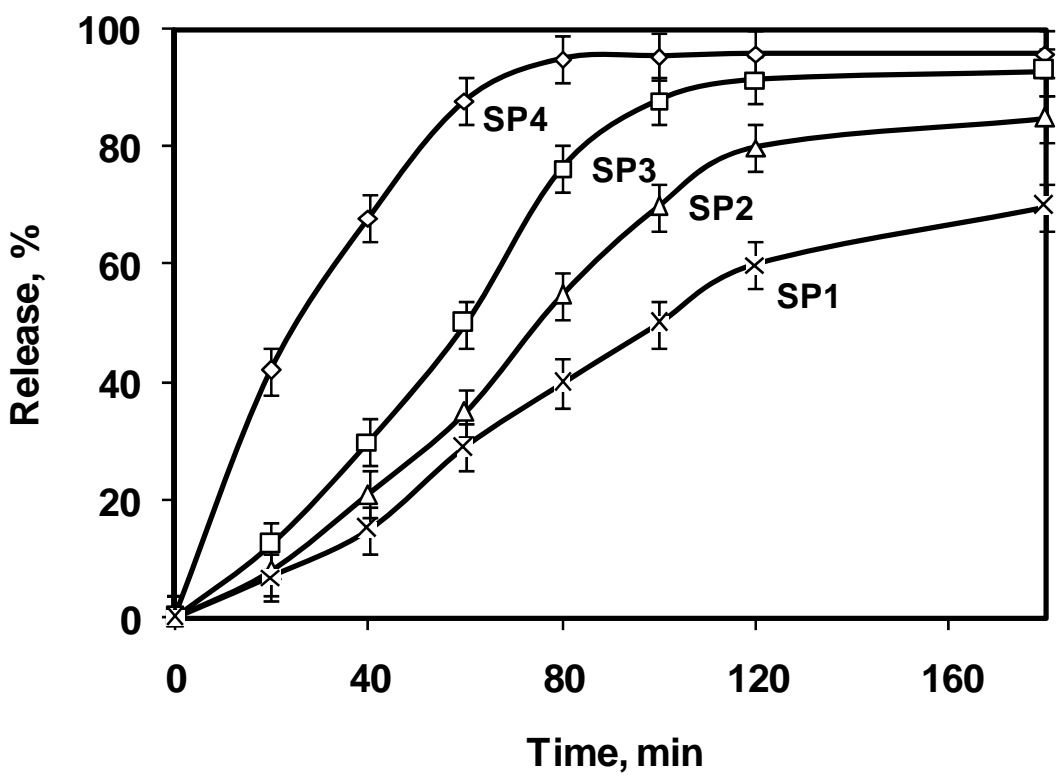

Fig. 6. Release dependency of hydrogels as a function of porosity. 
Secondly, as the porosity of the hydrogels increases, the slope of release begins to be sharper. Increasing the slope of release profile can be attributed to the fact that as the porosity of matrix is increased, the water uptake takes place faster, and subsequently the diffusion of the active agent through hydrogel is increased. As it is presented in Figure 6, after 80 minutes, only $40 \%$ of the active agent has been released from non-porous hydrogel, see SP1; while in the case of SP4 with high porosity, $95 \%$ of the active agent has been diffused into release medium. According to the results shown in Figure 6, the release rate of 2,4-D from hydrogel matrix is in agreement with dynamic swelling.

\section{Effect of MBA on the Release of 2,4-D}

To investigate the effect of MBA crosslinker on the release kinetics of 2,4-D, we have measured the percentage of release from hydrogels containing different amounts of crosslinker. The release percentage of the active agent is presented in Figure 7. The results indicate that the release of the active agent decreases as we increase the MBA concentration in the hydrogel matrix. When the crosslinker concentration is increased in hydrogel composition, the pore size decreases which causes: a) decrease in the loading content (Experimental section, Table 4) because of the low swelling capacity of hydrogels and b) the rate of diffusion of the active agent into release medium is decreased.

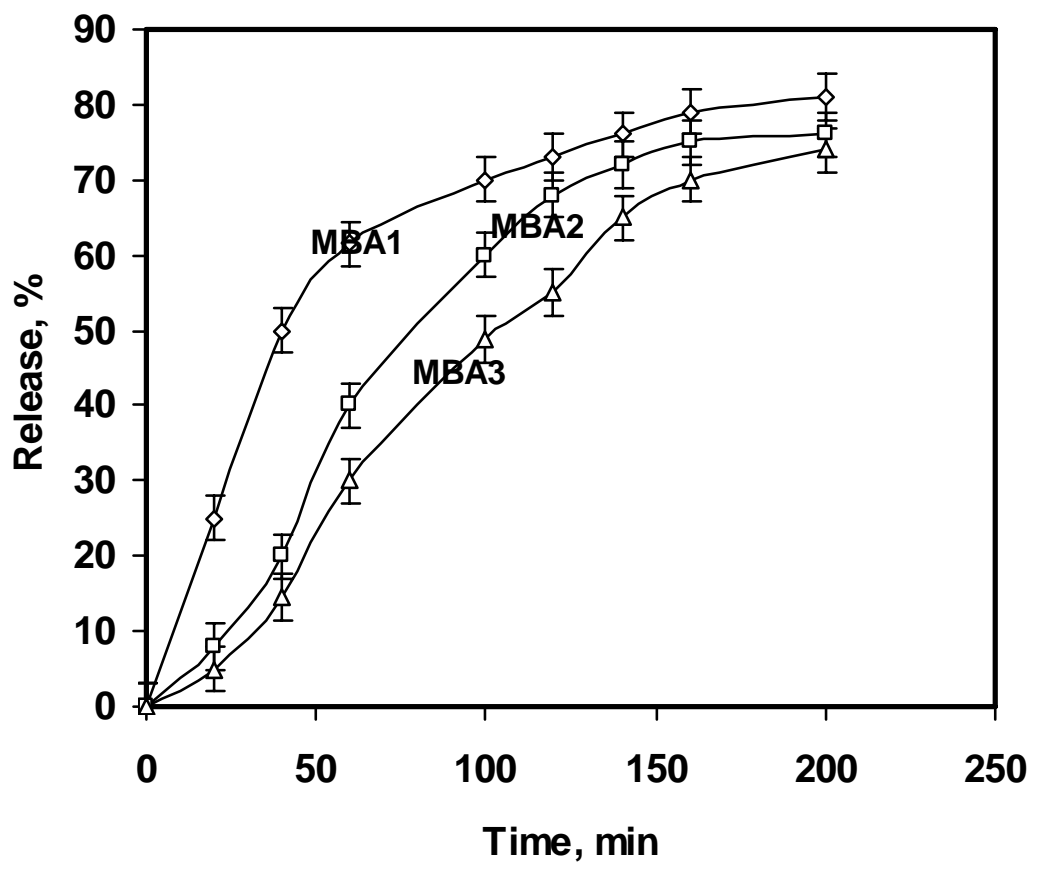

Fig. 7. Effect of MBA concentration on the release of 2,4-D.

Effect of $\mathrm{pH}$ on the Release of 2,4-D

Despite PAAm hydrogel is a non-ionic hydrogel, 2,4-D comprises carboxylic acid group and is known as an acidic compound. Therefore, the $\mathrm{pH}$ of the medium can affect the release of this active agent [33, 35]. The pKa value of 2,4-D is around 2.6. When the $\mathrm{pH}$ of the medium is lower than 2.6, the functional group of 2,4-D exists as 
a carboxylic acid $(-\mathrm{COOH})$ group. But, when the $\mathrm{pH}$ of the medium is higher than the pKa value, the carboxylic acid group will be converted to carboxylate (-COO'). Release dependency of 2,4-D as a function of $\mathrm{pH}$ is shown in Figure 8. As we discussed, the slow and low release of 2,4-D in $\mathrm{pH} 1$ is attributed to the non-ionic property of the active agent at low $\mathrm{pH}$.

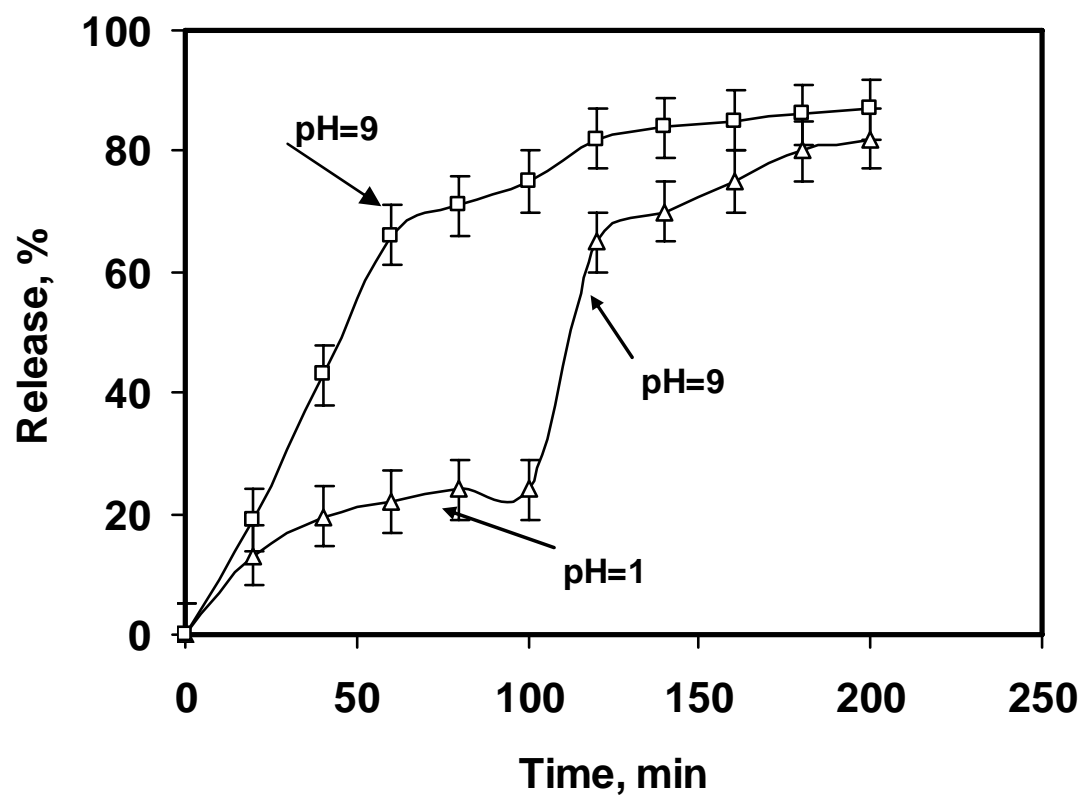

Fig. 8. Effect of $\mathrm{pH}$ on the release of 2,4-D.

The water solubility of 2,4-D is 311 and $43134 \mathrm{mg} / \mathrm{L}$ at pHs 1 and 9, respectively [36]. According to this fact, low release of 2,4-D can be attributed to a) protonation of the carboxylate group and subsequent conversion to a low soluble material and $b$ ) it may be attributed to the formation of hydrogen bonding between carboxylic acid group of 2,4-D and amid groups of PAAm chains (Scheme 2).

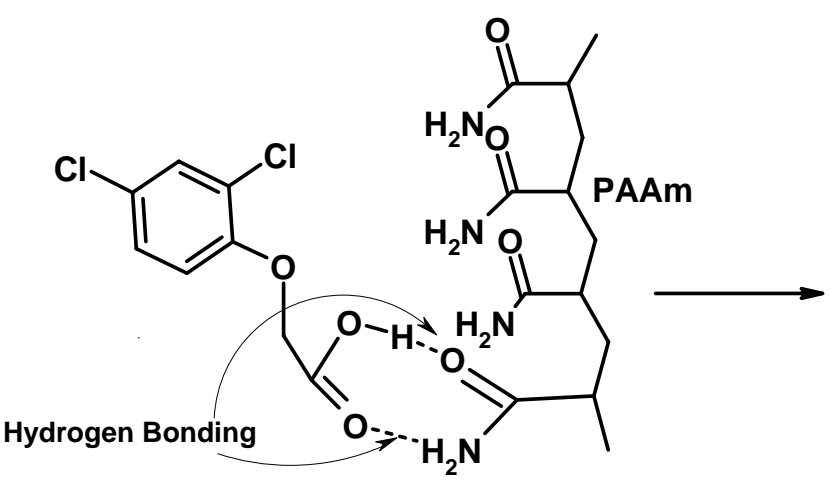

2,d-Dichlorophenoxyacetic Acid

pH 1<smiles>O=C([O-])COc1ccc(Cl)cc1Cl</smiles>

2,d-Dichlorophenoxyacetate<smiles>CC(CC(CC(CC(C)C(N)=O)C(N)=O)C(N)=O)C(N)=O</smiles>

pH 9

Scheme 2. Effect of $\mathrm{pH}$ on the behaviour of 2,4-D. 
Fast release of the active agent in basic solutions is due to the presence of anionic carboxylate groups. When the time of release reaches $100 \mathrm{~min}$, only $24 \%$ of the active agent was released into acidic solution, while in the basic medium, $75 \%$ of 2,4-D was diffused into the medium after the same amount of time passed. As shown in Figure 8, when the hydrogel is transferred from acidic to basic solution, the release of the active agent suddenly increases. This phenomenon is attributed to conversion of $\mathrm{COOH}$ to $\mathrm{COO}^{-}$. Thus, the active agent easily diffuses into the releasing medium.

\section{Conclusions}

Porous PAAm hydrogel was synthesized using calcium carbonate powders and it was subsequently removed from the medium by the technique already discussed. We have confirmed the porous structure of hydrogel using SEM technique. It also indicates that higher porosity is achievable using higher concentration of calcium carbonate. The swelling capacity of hydrogels was increased when the $\mathrm{CaCO}_{3}$ amount was increased in the preparation step, which is due to an increase in porosity and higher water uptake ability. On the contrary, increase in MBA concentration results in lower swelling ability due to denser crosslink and shrinkage in network space. The release of 2,4-D herbicide as a function of MBA concentration was investigated and results indicate that increased concentration of MBA has negative effect on release of the active agent. The release profiles at various $\mathrm{pH}$ showed $\mathrm{pH}$ dependency release of 2,4-D herbicide, i.e., we observed fast release of the active agent in basic solutions and slow release in solutions with $\mathrm{pH}$ lower than 2.6. We believe that the functional group, carboxylic acid, plays an important role in this phenomenon and causes this $\mathrm{pH}$ dependency.

\section{Experimental}

\section{Materials}

Acrylamide (AAm), Merck Co., was used after crystallization from acetone. MBA, APS and SMBS (Fluka Co.), were used as received. CCb $(\sim 3 \mu \mathrm{m})$, Fluka Co., and other chemicals were used as received. Herbicide 2,4-D (Sigma Aldrich Chemicals) was used as received.

\section{Synthesis of PAAm Hydrogel}

AAm solution was prepared in a one-liter reactor equipped with mechanical stirrer and gas inlet. AAm was dissolved in degassed distillated water. Reaction variables are given in Table 3 .

Tab. 3. Reaction conditions for synthesis of porous hydrogels $\left(\mathrm{H}_{2} \mathrm{O} \quad 40 \mathrm{~mL}\right.$, APS/SMBS 0.22/0.26 mmol, AAm $42 \mathrm{mmol}$ ).

\begin{tabular}{cccccccc}
\hline Run & SP1 & SP2 & SP3 & SP4 & MBA1 & MBA2 & MBA3 \\
\hline MBA, mmol & 0.32 & 0.32 & 0.32 & 0.32 & 0.16 & 0.32 & 0.65 \\
\hline $\mathrm{CaCO}_{3}, \mathrm{mg} / \mathrm{mL}$ & 0 & 38 & 75 & 150 & 75 & 75 & 75 \\
\hline
\end{tabular}


In general, AAm (3.0 g, $42 \mathrm{mmol}$ ) was dissolved in $30.0 \mathrm{~mL}$ of distillated degassed water. MBA as a crosslinker $(0.050 \mathrm{~g}$ in $2 \mathrm{~mL}$ water, $0.32 \mathrm{mmol})$ was added to the AAm solution and the mixture was continuously stirred under nitrogen gas. Various amount of calcium carbonate powder $(38,75,150 \mathrm{mg} / \mathrm{mL})$ was added to the reaction mixture and allowed to stir for $10 \mathrm{~min}$. Then APS $(0.05 \mathrm{~g}$ dissolved in $2 \mathrm{~mL}$ water, $0.22 \mathrm{mmol}$ ) and SMBS (0.05 g dissolved in $2 \mathrm{~mL}$ water, $0.26 \mathrm{mmol}$ ) as a redox initiator were added to the solution and stirred for $30 \mathrm{~min}$. Obtained hydrogels were cut in same sizes $(\sim 2 \times 2 \times 2 \mathrm{~mm})$. Subsequently, the calcium carbonate particles were dissolved in the aqueous $10 \% \mathrm{wt} \mathrm{HCl}$ solution by immersion of hydrogels for $24 \mathrm{~h}$ at ambient temperature. For removing the calcium chloride from dissolution of calcium carbonate and remaining substrates, the hydrogels were immersed in excess amount of deionized water for 6 days with daily refreshment of the water. In order to assure the complete removal of $\mathrm{Ca}^{2+}$ and $\mathrm{Cl}^{-}$ions, $\mathrm{CO}_{3}{ }^{2-}$ and $\mathrm{Ag}^{+}$ions were used, respectively; in which lack of any precipitation was attributed to a complete removal. After this time, the hydrogels were poured to the $300 \mathrm{~mL}$ ethanol. After dewatering for $24 \mathrm{~h}$, the hardened hydrogel product were filtered, washed with fresh ethanol $(2 \times 50$ $\mathrm{ml}$ ) and dried at $50{ }^{\circ} \mathrm{C}$ until obtaining a constant weight.

\section{Density Measurement}

In this section of study, the hydrogels were dried at $100{ }^{\circ} \mathrm{C}$ for $24 \mathrm{~h}$. Bulk density of dried hydrogels was determined by using picnometric method outlined in ASTM D792. In general, $1 \mathrm{~g}$ of dried hydrogels was placed in a 10-mL picnometer with known weight. Then the picnometer containing sample was filled with acetone as non-solvent and picnometer containing sample and acetone weighed to determine the density of sample according to standard method.

\section{Swelling Studies}

Dried hydrogel were used to determine the degree of swelling. The degree of swelling (DS) was determined by immersing the hydrogels $(0.1 \mathrm{~g})$ in distillated water $(100 \mathrm{~mL})$ and it was allowed to soak for $24 \mathrm{~h}$ at room temperature. Then, they were removed from the water, blotted with filter paper to remove surface water, weighed and the DS was calculated using Eq (7):

$D S=\left(W_{s}-W_{d}\right) / W_{d}$

where $W_{s}$ and $W_{d}$ are the weights of the samples swollen in water and in dry state, respectively. For studying the swelling kinetics of the hydrogels, a certain amount of samples $(0.20 \mathrm{~g})$ was poured into several weighed tea bags and was immersed in $200 \mathrm{ml}$ distilled water. At consecutive time intervals, the water absorbency of the hydrogels was measured according to the above mentioned method.

\section{Loading/Release of 2,4-D}

The 2,4-D was dissolved in $25 \mathrm{ml}$ deionized water with desired concentration $(0.2$ $\% w t)$. An important note is that the sodium salt of 2,4-D was used for loading. For this purpose, we used stochiometric amount of $\mathrm{NaOH}$ to dissolve it. Afterward, we immersed pre-weighed purified and dried hydrogel $(0.1 \mathrm{~g})$ into the prepared solution for $24 \mathrm{~h}$ at room temperature. Loaded hydrogels were filtered and dried in ambient temperature to take them to constant weight (The 2,4-D was measured using UVVisible spectrometer at $\lambda_{\max }=284 \mathrm{~nm}$ ). For estimation of the percentage of loaded herbicide, the loaded hydrogels were immersed in deionized water for $24 \mathrm{~h}$ and 
released herbicide after $24 \mathrm{~h}$ was assumed as loaded 2,4-D. Using calibration curve, the released 2,4-D was calculated. Table 4 shows the percentage of loading for hydrogels.

Tab. 4. The percentage of loading of 2,4-D for hydrogels.

\begin{tabular}{cccccccc}
\hline Sample & MBA1 & MBA2 & MBA3 & SP1 & SP2 & SP3 & SP4 \\
\hline \%Loading & 8.5 & 6.5 & 3.5 & 3.8 & 5.5 & 7 & 9 \\
\hline
\end{tabular}

Deionized water was chosen as a release medium to study the percentage release of 2,4-D. In general, dried 2,4-D loaded hydrogels were immersed in $100 \mathrm{~mL}$ deionized water under un-stirred condition. At intervals, the amount of released 2,4-D was evaluated using UV spectrometer. The percentage of released 2,4-D was determined from the calibration curve.

\section{Instruments}

The surface morphology of the dried hydrogels was examined using scanning electron microscopy (SEM). Dried hydrogels were ground and sieved to 40-60 mesh and used for SEM studying. Dried hydrogel powders were coated with a thin layer of palladium gold alloy and imaged in a SEM instrument (Leo, 1455 VP). An UV-Visible (UNICO, 2102 Series) was used to study release of 2,4-D.

\section{Acknowledgements}

The authors would like to thank the President of University of Maragheh, Dr. Gholam Reza Hojjati, and Research Deputy of University of Maragheh for their support to carry out this project. Also, the authors would like to specially thank the referees for their suggestions.

\section{References}

[1] Po, R. J.M.S.-Rev. Macromol. Chem. Phys. 1994, C34, 607.

[2] Sdayal, U.; Mehta, S.K.; Choudhary, M.S.; Jain, R.C. J.M.S.-Rev. Macromol. Chem. Phys. 1999, C39, 507.

[3] Liu, Q.; Rauth A.M.; Wu, X.Y. Int. J. Pharm. 2007, 339, 148.

[4] Tada, D.; Tanabe,T.; Tachibana, A.; Yamauchi, K. J. Biosci. Bioeng. 2005, 100, 551.

[5] Liu, M.; Liang, R.; Zhan, F.; Liu, Z.; Niu, A. Polym. Adv. Technol. 2006, 17, 430.

[6] Bajpai, A.K.; Giri, A. React. Funct. Polym. 2002, 53, 125.

[7] Naganagouda, N.K.; Mulimani, V.H. Process Biochem. 2006, 14, 1903.

[8] Sheldon, R.A. Adv. Synth. Catal. 2007, 349, 1289.

[9] Chen, J.; Park, K. J. Control. Release 2000, 65, 73.

[10] Omidian, H.; Rocca, J.G.; Park, K. J. Control. Release 2005, $102,3$.

[11] Xiao, H.; Yue, Z.; Henry, J.D.; Tao L.L. Tissue Eng., 2007, 13, 2645.

[12] Lu, G.D.; Yan, Q.Z.; Ge, C.H. Polym. Int. 2007, 56, 1016.

[13] Omidian, H.; Rocca, J.G.; Park, K. Macromol. Bioscience 2006, 6, 703.

[14] Caykara, T.; Bulut, M.; Dilsiz, N.; Akyuz, Y. J. Macromol. Sci. Part A: Pure and Appl. Chem. 2006, 43, 889.

[15] Thomas, W.M.; in Acrylamide Polymers, Ed. Bikales NM. Encyclopedia of Polymer Science and Technology, Vol. 1, New York, Wiley (1964).

[16] Saraydin, D.; Karadag, E.; Oztop, N.; Guven, O. Biomaterials 1994, 15, 917. 
[17] Saraydin, D.; Karadag, E.; Cetinkaya, S.; Guven, O. Radiat. Phys. Chem. 1995, 46, 1049.

[18] Abd El-Rehim , H.A. J. Appl. Polym. Sci. 2006, 101, 3572.

[19] Abd El-Rehim, H.A.; Hegazi, E.A.; Abd El-Mohdy, H.L. J. Appl. Polym. Sci. 2005, 98, 1262.

[20] Kandil, S.; Kenawy, R.; El-Maghraby, A.; Elashry, E.H. J. Appl. Polym. Sci. 2004, $94,1420$.

[21] Piletska, E.V.; Turner, N.W.; Turner, A.P.F.; Piletsky, S.A. J. Controlled Release 2005, 108, 132.

[22] Zhuo, Z.; Zhuo, R. Eur. Polym. J. 2001, 37, 1913.

[23] Moreno, L.D.; Moreno, L.S.; Pena, A. Environment 2007, 378, 119.

[24] Hermosin, M.C.; Calderon, M.J.; Aguer, J.P.; Cornejo, J. Pest Manag. Sci. 2001, $57,803$.

[25] Garcia, M.F.; Chaves, M.S. J. Appl. Polym. Sci. 2006, 102, 4238.

[26] Zhu, Z.; Zhuo, R. Starch/Starke 2000, 52, 58.

[27] Zhu, Z.; Zhuo, R. J. Appl. Polym. Sci. 2001, 81, 1535.

[28] William, J.; Connick, Jr. J. Appl. Polym. Sci. 1982, 27, 3341.

[29] Richard, A.G.; Park, H.; Park, K. Polym. Adv. Technol. 2000, 11, 617.

[30] Tamagawa, H.; Popovic, S.; Taya, M. Polymer, 2000, 41, 7201.

[31] Wu, J.; Lin, J.; Zhou, M.; Wei, C. Macromol. Rapid. Commun. 2000, 21, 1032.

[32] Omidian, H.; Hashemi, S.A.; Sammes, P.G.; Meldrum, I. Polymer 1998, 39, 6697.

[33] Tatavarti, A.S.; Mehta, K.A.; Augsburger, L.L.; Hoag, S.W. J. Pharm. Sci. 2004, 93, 2319.

[34] Thill, D. Growth regulator herbicides p.267-291. In: Herbicide action course. West Lafayette: Purdue University (2003).

[35] Gabr, K. Eur. J. Pharm. Biopharm. 1992, 38, 199.

[36] Hopkins, D. 2,4-Dichlorophenoxyacetic acid: Determination of the water solubility: Lab project Number: ES/DR/0002/2297/8. Dow Chemical Co., Unpublished (1987). 